\title{
Secondary Relaxation inside the Glass
}

\author{
Soheil Sharifi' ${ }^{1}$ and Jahanbakhsh Mashaiekhy Asl' ${ }^{2}$ \\ ${ }^{1}$ Department of Physics, University of Sistan and Baluchestan, P.O. Box 98155-987, Zahedan, Iran \\ ${ }^{2}$ Iranian National Center of Laser Science and Technology, P.O. Box 1465733441, Tehran, Iran
}

Correspondence should be addressed to Soheil Sharifi, soheil.sharifi@gmail.com

Received 5 April 2011; Accepted 18 May 2011

Academic Editors: H. Rahier and Y. Yue

Copyright ( 2011 S. Sharifi and J. Mashaiekhy Asl. This is an open access article distributed under the Creative Commons Attribution License, which permits unrestricted use, distribution, and reproduction in any medium, provided the original work is properly cited.

The physical properties of the glass depend on the procedure used to produce the glass. In particular, if the glass is obtained through the variation of external thermodynamic parameters, the specific way in which the parameters are varied (thermodynamic history) has influence on the final properties. In this work, we studied the effect of thermodynamic history on secondary relaxation inside the glassy state on different molecular glass forming, namely, PPGE(poly[(phenyl glycidyl ether)-co-formaldehyde]), 1,18-bis (p methoxyphenyl) cyclohexane (BMPC), poly(propylene glycol)—(PPG400), phenolphthalein-dimethyl-ether(PDE), Poly(vinyl acetate) (PVAc), and poly(bisphenol A-co-epichlorohydrin) glycidyl end-capped (DGEBA). We found secondary relaxation change with thermodynamic history and depends on the value of the activation volume which activation entropy of secondary relaxation inside the glassy state. Also, we found most of the JG secondary relaxation change with thermodynamic history and most of the Non-JG secondary relaxation are not sensitive.

\section{Introduction}

The state of the glass depends on the procedure used to produce the glass. In particular, if the glass is obtained through the variation of any external thermodynamic parameters, the specific way in which the parameter is varied (thermodynamic history) influences the molecular arrangement. For example, different combinations of pressure and temperature variations can be used, or only varying temperature, different cooling rates can be applied to the supercooled liquid. A further consequence is that thermodynamic properties such as enthalpy, volume, and so forth, also depend on the thermodynamic history. In addition, dynamic processes can change with the different molecular arrangement frozen in the glass. For example, they present different values of relaxation time and different intensity when the glass is prepared through different procedures. This phenomenon is well known for the structural relaxation measured just below the glass transition temperature, $T_{g}$, after different cooling rates [1]. The secondary relaxation was studied in several glassy systems, a study on $\mathrm{GeO}_{2}\left(\mathrm{HQGeO}_{2}\right)$ glass, that is, one of the strongest glass systems and showed that the quantitative relationship between activation energy of secondary relaxation $\left(E_{\beta}\right)$ and $T_{g}$ agrees well with the empirical relation of the Johari-Goldstein relaxation, [2], also a study on the secondary $(\beta)$ relaxation in $\mathrm{La}_{55} \mathrm{Al}_{25} \mathrm{Ni}_{20}$ metallic glasses showed a relation between activation energy of secondary relaxation glass transition temperature, [3].

As previously introduced, secondary relaxation processes are the only process active in the deep glassy state. For a long time, secondary processes were believed to reflect only simple and localized motion of the molecule, which should not be influenced by the molecular arrangement. Instead, it was recently evident that some secondary relaxation depend upon the thermodynamic history by which the glass is formed [4]. Many experiments were performed testing the effect of different cooling rates on secondary processes, and usually a dependence on the relaxation strength and maximum frequency of the secondary process are observed both for intermolecular origin Johari-Goldstein (JG) and intramolecular secondary processes (non-JG relaxation), [5]. Secondary relaxation originates from different types of motions: localized fluctuation of the whole molecules, or the rotational fluctuations of the side groups or parts of them. When motion depends on whole molecule it is designated as the Johari-Goldstein (JG) process. In another case, the investigation was performed at high-pressure, using different combinations of pressure and temperature variations. For 
example, a study about diisobutyl phthalate showed that the characteristic relaxation time changes when measured after different thermodynamic history. Dielectric loss curves of the secondary relaxation of diisobutyl phthalate are reported as measured for the same value of pressure and temperature, but after that, the glass was prepared through different thermodynamic paths. It is readily apparent that the various pathways yield very different frequencies for the maximum in the secondary relaxation process [5]. In this study, we present experimental studies varying pressure and temperature of secondary processes in several glass formers. We present the study of the influence of thermodynamic history on the secondary process of different molecular glass formers, namely PPGE (poly[(phenyl glycidyl ether)-co-formaldehyde]), 1,18-bis (p methoxyphenyl) cyclohexane (BMPC), poly (propylene glycol)_(PPG400), phenolphthalein-dimethyl-ether (PDE), Poly(vinyl acetate) (PVAc), and Poly(Bisphenol A-co-epichlorohydrin) glycidyl end-capped (DGEBA). All of these systems present a complex relaxation scenario, with more than one secondary process. In this research, we focused on the slower secondary process of BMPC (non-JG relaxation), PDE (non-JG relaxation), DGEBA (JG relaxation) and PPGE (JG relaxation), the faster of PPG400 (non-JG relaxation) and the only one observed in and PVAc, whose microscopic origin is not clear. In such a way we have the possibility of studying the effect of thermodynamic history on secondary processes of JG type (PPGE, DGBEA), as well as of intramolecular origin (non-JG relaxation) (PDE, BMPC, PPG400).

\section{Experiment and Materials}

Poly[(phenyl glycidyl ether)-co-formaldehyde] (PPGE) with average molecular weight, M.W. $=345 \mathrm{~g} / \mathrm{mol}, T_{g}=258 \pm$ $1 \mathrm{~K}$, was purchased from Aldrich. Glassy PPGE shows two secondary processes, the slower being of JG type and the faster probably related to local motion of the epoxy subunits, [6-12]. Phenolphthalein-dimethylether (PDE), with M.W. $=346 \mathrm{~g} / \mathrm{mol}$, was synthesised in the laboratory of professor H. Sillescu and obtained from Professor M. Paluch. The glass transition temperature of PDE at atmospheric pressure is about $298 \mathrm{~K}$, and $T_{g}$ was observed to vary nearly linearly with pressure with a coefficient of about $0.26 \mathrm{~K} / \mathrm{MPa}$ (up to $200 \mathrm{MPa}$ ) PDE sample. Glassy PDE presents three different relaxation processes: so-called excess wing, and two secondary relaxation processes. The excess wing reflects the JG relaxation, whereas both the secondary processes reflect local motions of parts of the molecule [1318]. 1,18-bis (pmethoxyphenyl) cyclohexane (BMPC) with average molecular weight, M.W. $=296 \mathrm{gr} / \mathrm{mol}$, and $T_{g}$ at ambient pressure around $246 \pm 1 \mathrm{~K}$, was synthesised in the laboratory of professor H. Sillescu and obtained from Professor M. Paluch. Glassy BMPC showed two secondary relaxations whose molecular origin is not clear at all, $[17,18]$. Poly(propylene glycol)-(PPG400) with average molecular weight M.W. $=400 \mathrm{~g} / \mathrm{mol}$, was purchased from Fluka. In glassy PPG400, we observe two secondary relaxations, the slower was identified as the true JohariGoldstein relaxation [19], whereas the faster is supposed to originate from intramolecular motions. Poly(Bisphenol A-co-epichlorohydrin), glycidyl end-capped (DGEBA) with average molecular weight $\sim 380 \mathrm{~g} / \mathrm{mol}$ and glass transition temperature $255 \pm 1 \mathrm{~K}$, was purchased from Shell Co. under the trade name of Epon 828. In the glassy state, two secondary processes are present, and the slower one was identified as the JG process [20-22]. Poly(vinyl acetate) (PVAc) with M.W. $=167 \mathrm{Kg} / \mathrm{mol}$, and $T_{g}=310.7 \mathrm{~K}$, is purchased from Aldrich. PVAc has interesting structural variations and can be obtained in the atactic, and thus amorphous form, which is crucial for investigations of the glass transition. PVAc has been often chosen to test the current aspects of various theories related with glass transition phenomena. An ample dipole moment makes PVAc a good candidate of dielectric spectroscopy [23]. Dielectric measurements were carried out by a dielectric spectrometer $\left(\alpha\right.$-Novocontrol) in the frequency interval $10^{-2}-10^{7} \mathrm{~Hz}$. For measurements at ambient pressure, the sample was placed in a parallel plate cell (diameter $30 \mathrm{~mm}$, gap $0.1 \mathrm{~mm}$ ) and the temperature control was performed with a precision better than $0.1 \mathrm{~K}$ by using a dry nitrogen stream-based system. For measurements at high-pressure, the sample was placed in a parallel plate cell (diameter $20 \mathrm{~mm}$, gap $0.05 \mathrm{~mm}$ ) that, properly insulated from the external environment, was located inside a pressure chamber. Pressure variations (0.1-600 MPa) were generated by a manual pump and transmitted to the sample through silicon oil. A liquid circulator connected to a jacket, wrapped around the pressure room, allowed the control of temperature (353-233 K) within $0.1 \mathrm{~K}$.

\section{Results and Discussion}

Representative isothermal dielectric loss spectra of the investigated glass formers in the glassy state are presented in Figure 1. We acquired dielectric spectra by varying pressure from $0.1 \mathrm{MPa}$ up to the maximum value of $600 \mathrm{MPa}$. For all the systems, we can observe a peak corresponding to the secondary relaxation: in the following this relaxation will be named as $\beta$-relaxation with the exception for PPG400 that, in agreement with the literature, will be called $\gamma$-relaxation. In the case, of PPGE, DGEBA, and PDE we observe at high frequencies a rise in the signal partly due to the presence of a faster relaxation, which however is never completely visible with our apparatus for high-pressure measurements. In the case of BMPC and PPG400 at low frequencies, the tail of the structural peak is still present in some spectra. The $\beta$ relaxation in PPGE, DGEBA, PDE, and PVAc moves to lower frequencies faster than the secondary relaxation in BMPC and PPG400, Figure 1. It is reported that the $\beta$-secondary process of PPGE and DGEBA is of the JG type [24, 25], whereas the $\beta$-process of PDE and BMPC and the $\gamma$-process of PPG400 are non-JG $[26,27]$. We analyzed spectra in terms of a Havriliak and Negami (HN) function for the structural process, (1), and a Cole-Cole equation $(\beta=1$ at (1)) for the secondary relaxation

$$
\varepsilon(\omega)=\varepsilon_{\infty}+\frac{\Delta \varepsilon}{\left[1+\left(i \omega \tau_{\beta}\right)^{1-\alpha}\right]^{\beta}} .
$$




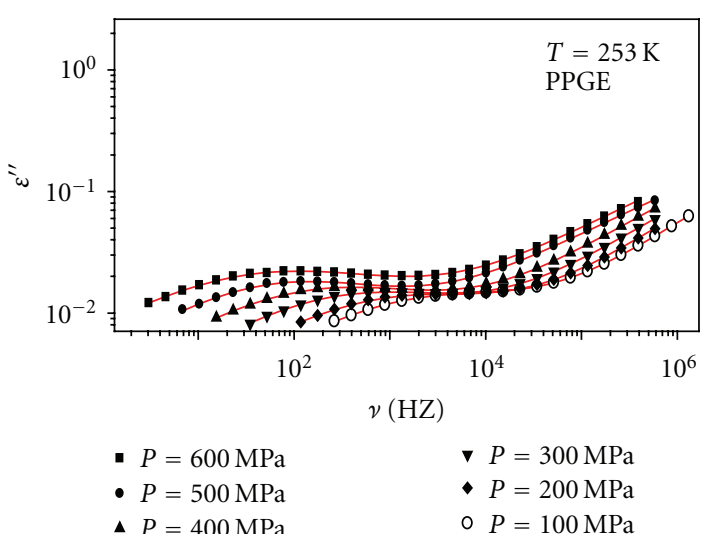

(a)

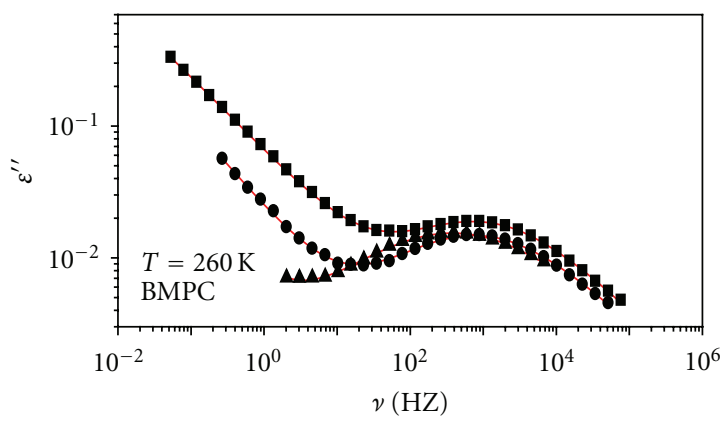

- $P=100 \mathrm{MPa}$

- $P=200 \mathrm{MPa}$

\ $P=500 \mathrm{MPa}$

(c)

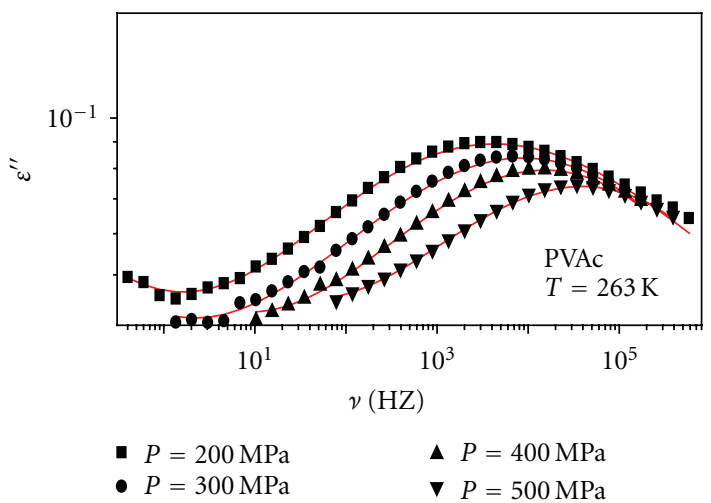

(e)

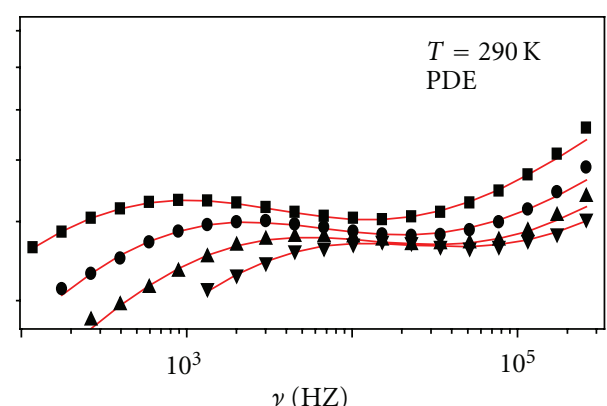

、 $P=250 \mathrm{MPa} \quad$ - $P=500 \mathrm{MPa}$

\ $P=400 \mathrm{MPa}$ - $P=600 \mathrm{MPa}$

(b)

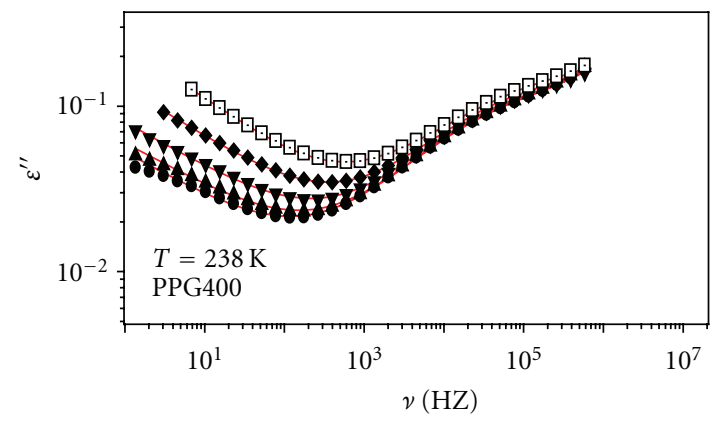

๑. $P=390 \mathrm{MPa}$

\ $P=480 \mathrm{MPa}$

- $P=420 \mathrm{MPa}$

- $P=515 \mathrm{MPa}$

v $P=453 \mathrm{MPa}$

(d)

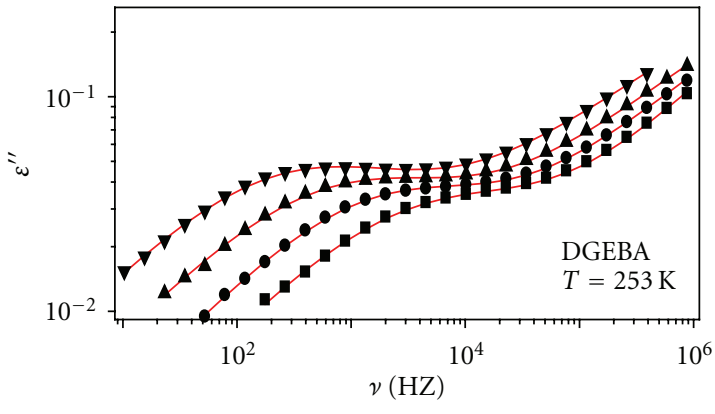

- $P=150 \mathrm{MPa}$

\ $P=350 \mathrm{MPa}$

- $P=250 \mathrm{MPa}$

V $P=450 \mathrm{MPa}$

Figure 1: Isothermal loss spectra of (a) PPGE, (b) PDE, (c) BMPC, (d) PPG400, (e) PVAc, and (f) DGBEA at various pressures in the glassy state. We can see the secondary peak that slightly moves with pressure.

In the cases of PPGE, PDE, and DGBEA, we added an $\mathrm{HN}$ equation for fitting the faster secondary relaxation $(\gamma$ relaxation). The shape parameters used in the $\mathrm{HN}$ equation for the $\gamma$-relaxation in these three systems were obtained from fitting of spectra at very low temperature and ambient pressure where the $\gamma$-relaxation can be clearly observed. Moreover, a similar procedure was used for fitting the tail of the structural peak when present in the spectra.
The pressure dependence of secondary relaxation for different materials with different origins is represented in Figure 2. The maximum frequency was calculated by, $v_{m}=$ $\left(1 / 2 \pi \tau_{\mathrm{cc}}\right)$, where $\tau_{\mathrm{cc}}$ is the parameter from fit with C.C. function.

The logarithm of the frequency of maximum decreases linearly with increasing pressure for all the systems. This dependence can be described by the pressure counterpart of 


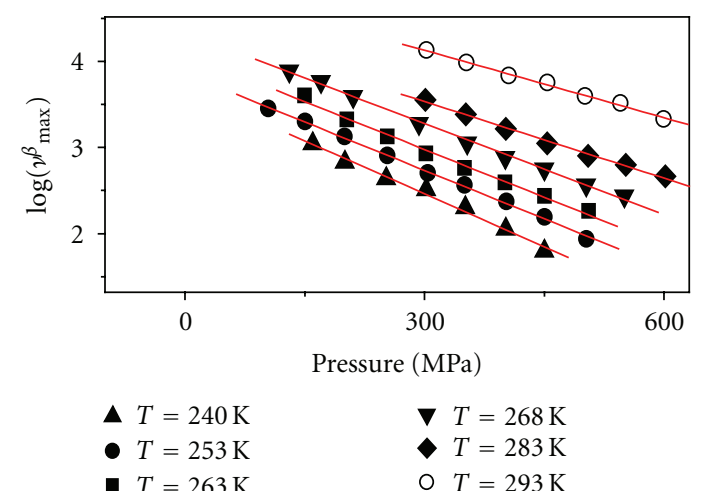

(a)

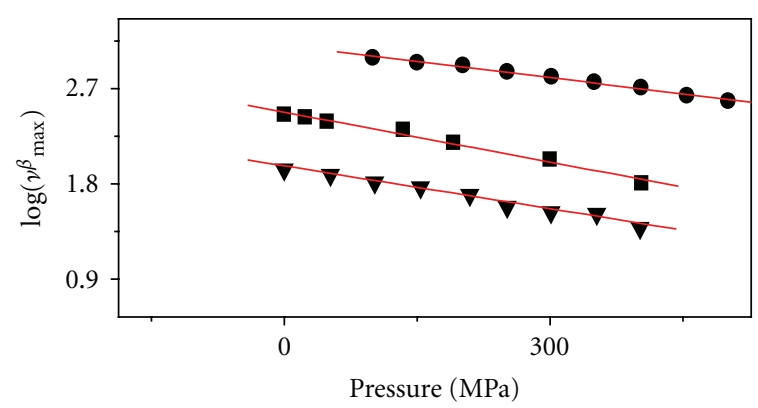

- $T=260 \mathrm{~K}$

a $T=246 \mathrm{~K}$

$\boldsymbol{\nabla} T=233 \mathrm{~K}$

(c)

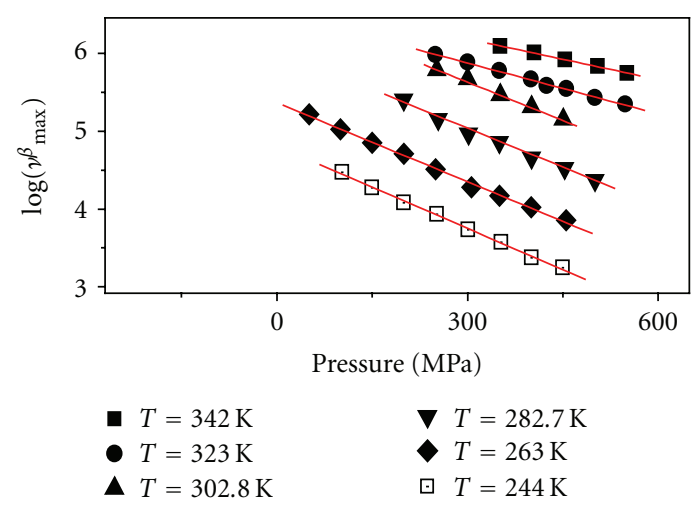

(e)

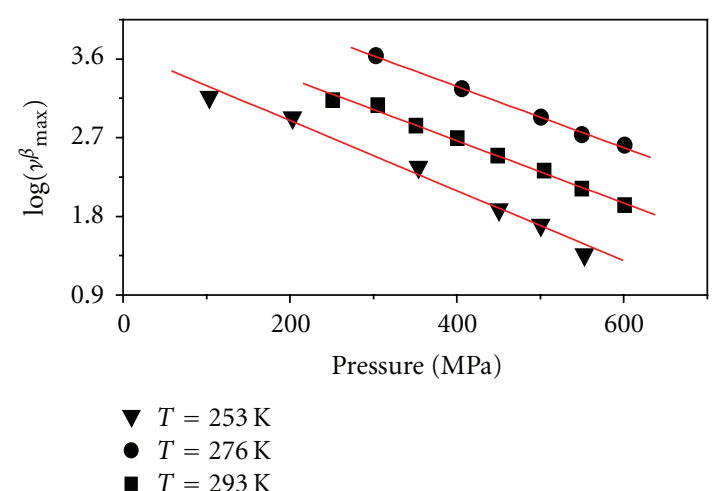

(b)

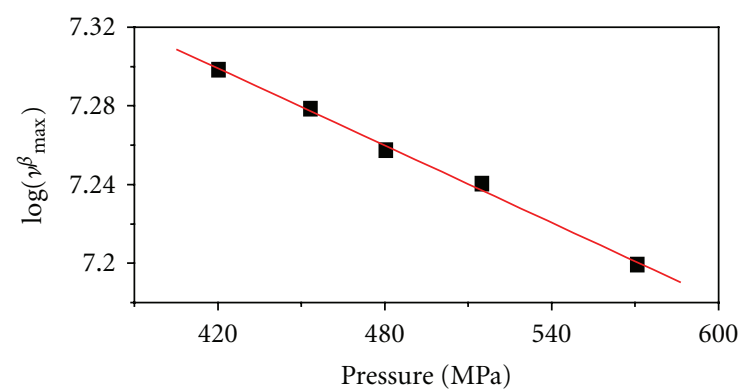

a $T=238 \mathrm{~K}$ (d)

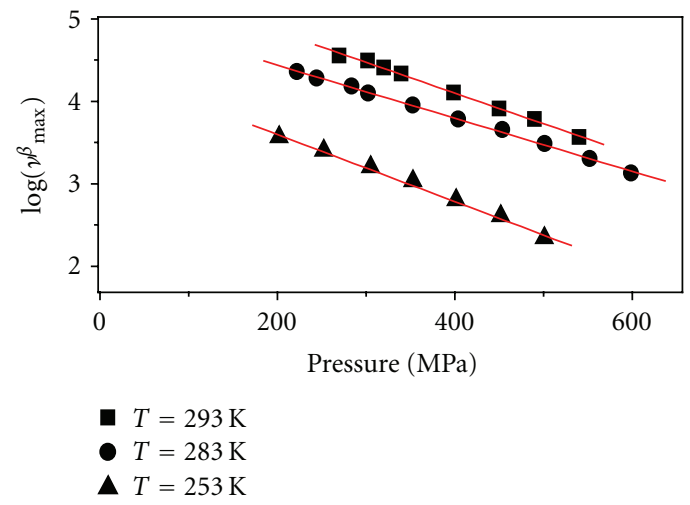

(f)

FIgURE 2: Frequency of the maximum of the dielectric loss peak of secondary relaxation as a function of pressure at different temperatures (as indicated in the figure) for (a) PPGE, (b) PDE, (c) BMPC, (d) PPG400, (e) PVAc, (f) DGBEA.

the temperature Arrhenius law, equation (2), [28-30]

$$
\Delta V_{\beta}=\ln (10) \cdot k_{B} T \cdot\left(\frac{d \log _{10} \nu_{\max }^{\beta}}{d P}\right)_{T} .
$$

By the analysis of the pressure dependence of the relaxation frequency, we obtained the values of activation volume $\Delta V_{\beta}$, Table 1.

The effect of temperature on secondary relaxation is presented in Figure 3. The analysis of dielectric spectra below
$T_{g}$ was performed using the same procedure as described in the previous paragraph.

The maximum frequency of secondary peak is reported in Figure 4, as a function of temperature for the different systems. We reproduced such behaviour with equations, namely, (3)

$$
\tau_{\beta}(T)=\frac{1}{2 \pi \nu_{\max }}=\frac{k_{B} T}{h} \exp \left(-\frac{\Delta S_{\beta}}{R}\right) \exp \left(\frac{\Delta H_{\beta}}{R T}\right),
$$




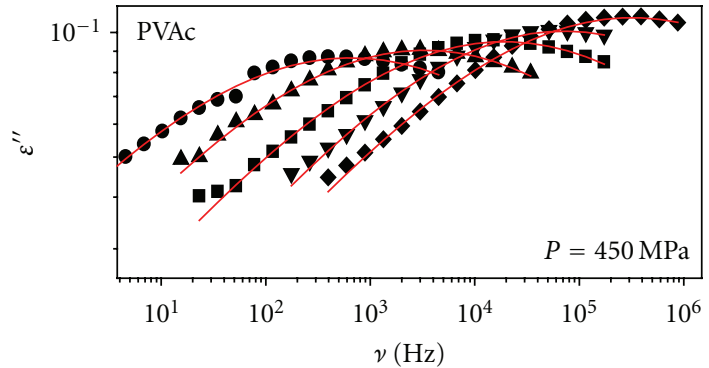

- $T=323 \mathrm{~K}$

$\boldsymbol{\nabla} T=303 \mathrm{~K}$

- $T=283 \mathrm{~K}$

(a)

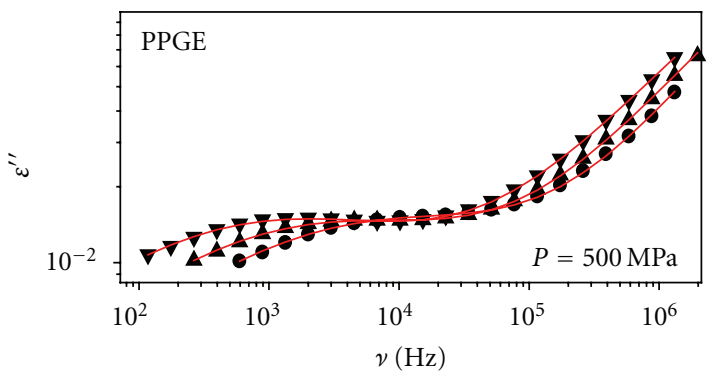

- $T=302.7 \mathrm{~K}$

$\Delta \quad T=292.7 \mathrm{~K}$

ק $T=282.9 \mathrm{~K}$

(c)

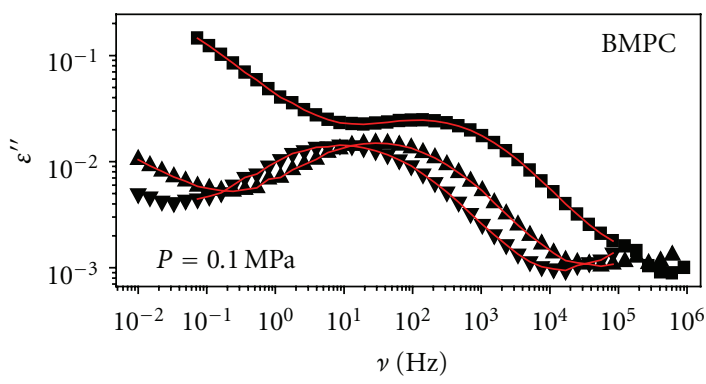

- $T=243 \mathrm{~K}$

$\Delta T=223 \mathrm{~K}$

$\boldsymbol{\nabla} T=213 \mathrm{~K}$

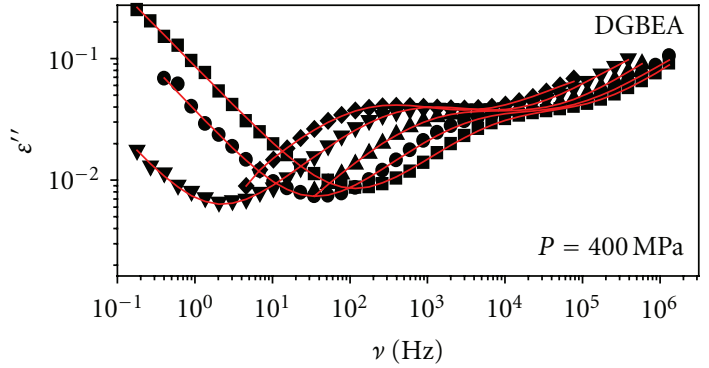

- $T=293.7 \mathrm{~K} \quad \boldsymbol{\nabla} T=258.3 \mathrm{~K}$

- $T=272 \mathrm{~K}$

$\Delta T=263.3 \mathrm{~K}$

(b)

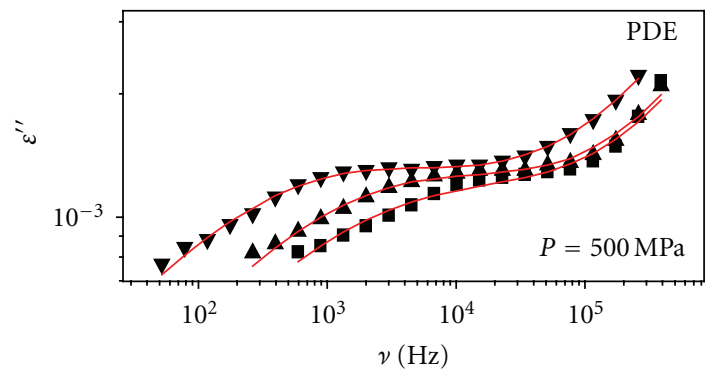

- $T=312.9 \mathrm{~K}$

А $T=302.8 \mathrm{~K}$

$\boldsymbol{\nabla} T=283.4 \mathrm{~K}$

(d)

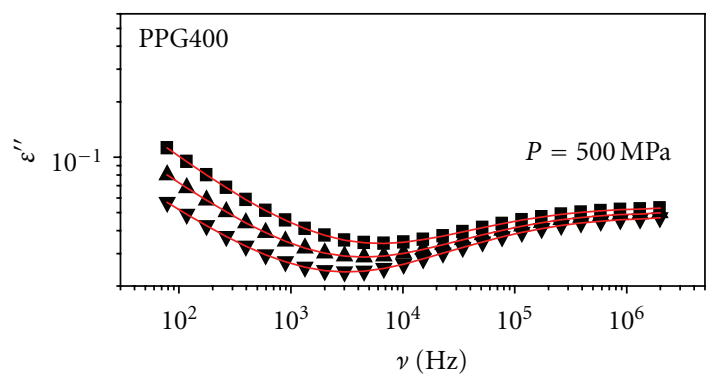

- $T=256 \mathrm{~K}$

$\Delta T=255 \mathrm{~K}$

$\boldsymbol{\nabla} T=254 \mathrm{~K}$

(e)

(f)

Figure 3: Isobaric loss spectra of (a) PVAc, (b) DGBEA, (c) PPGE, (d) PDE, (e) BMPC and (f) PPG400 at various temperatures in the glassy state.

where $\tau_{\beta}(T)$ is the temperature dependence of the relaxation time, $\Delta H_{\beta}$ and $\Delta S_{\beta}, k_{B}$ and $h$ represent, activation enthalpy and entropy of secondary relaxation, the Boltzmann constant, and the Plank constant. We found the activation entropy, $\Delta S_{\beta}$, and activation enthalpy, $\Delta H_{\beta}$, from Eyring equation (3). The obtained parameters are reported in Table 2.

Usually, both activation energy and enthalpy barriers increase with pressure, whereas activation entropy is almost insensitive or even sometimes decreases. In the case of PDE, activation energy decreases with the increase of pressure that is not clear but it may depend on the thermodynamic history used to prepare the glassy PDE. The behaviour of these parameter evidences the expected trend that the increase of density and the reduction of free volume hinder the motions related to the secondary relaxation, even when this is of intramolecular origin.

For studying the effect of thermodynamic history on secondary relaxations, we investigated glasses produced at the same values of external thermodynamic parameters but 


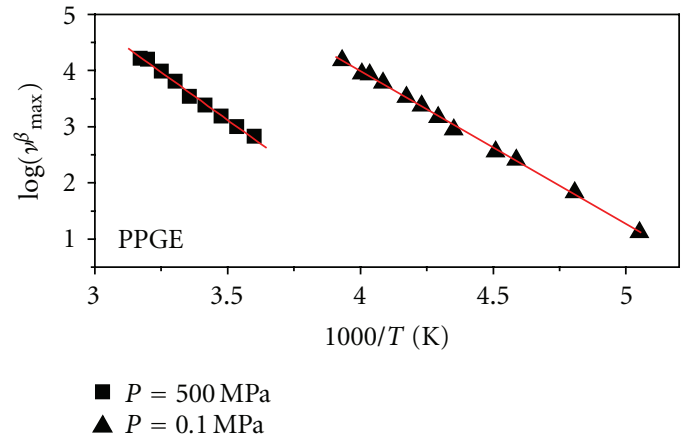

(a)

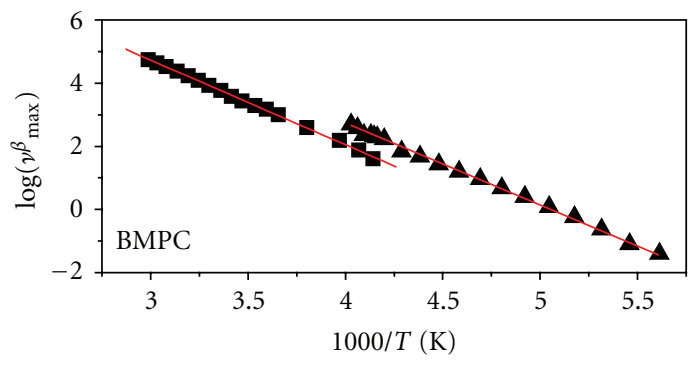

- $P=500 \mathrm{MPa}$

А $P=0.1 \mathrm{MPa}$

(c)

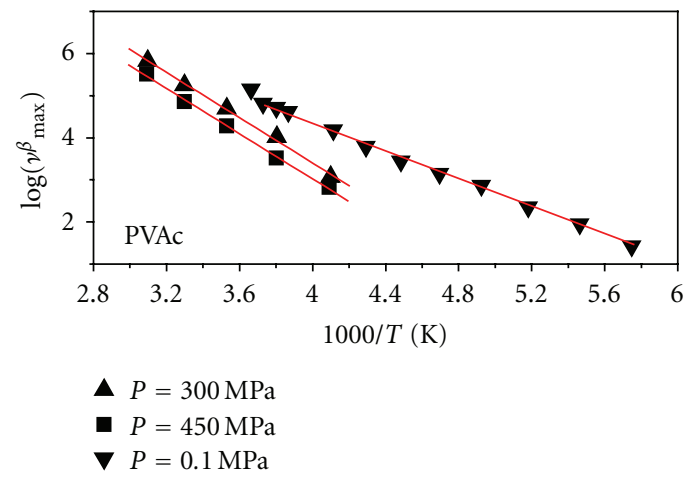

(e)

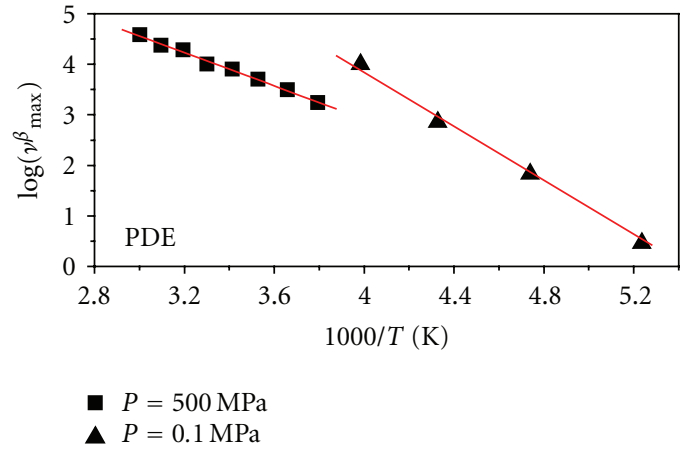

(b)

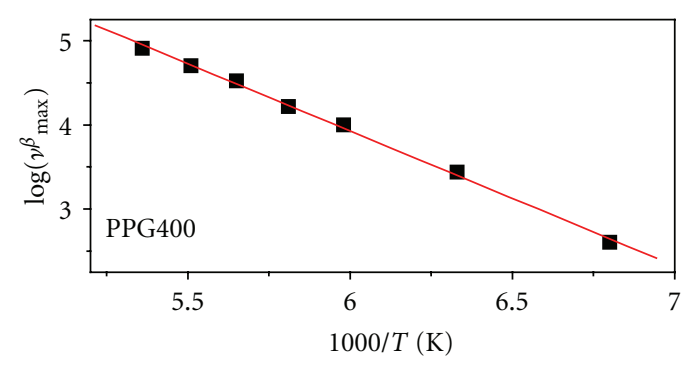

- $P=0 \mathrm{MPa}$

(d)

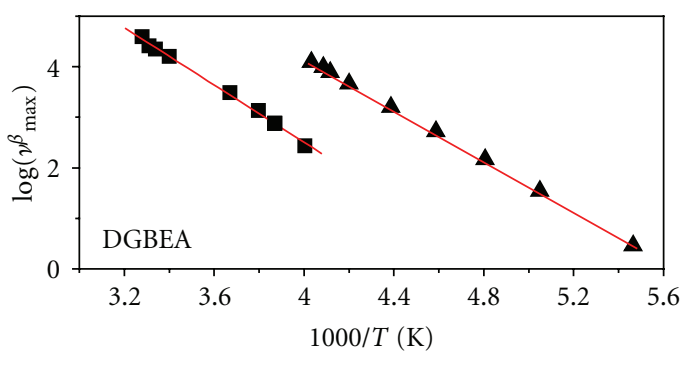

- $P=400 \mathrm{MPa}$

- $P=0.1 \mathrm{MPa}$

(f)

FIGURE 4: Frequency of the maximum in the dielectric loss as a function of pressure at different temperatures (as indicated in the figure) for (a) PPGE, (b) PDE, (c) BMPC, (d) PPG400, (e) PVAc, and (f) DGBEA. Red line is fit with Eyring equation (3).

obtained through different thermodynamic paths. For each material, we started the experiment from a thermodynamic point $\left(T_{i}, P_{i}\right)$, with changing the pressure and temperature along two different paths we got final points $\left(T_{f}, P_{f}\right)$, Figure 5. In the first path $\mathrm{A}$, we pressurized the liquid isothermally to the final pressure $P_{f}$ (at a temperature $T_{i}$ above $T_{g}$ at ambient pressure), and then we cooled it down to $T_{f}$ at constant pressure. In the second path $\mathrm{B}$, we isobarically cooled the system from $T_{i}$ to $T_{f}$, and then we increased pressure isothermally to $P_{f}$. Each material with the exception of PPG400 was vitrified by compression along the path A and by cooling along the path B. The estimated values of temperature and pressure at which materials were vitrified, together with the parameters characterizing the different paths for each material are reported in Table 3. The cooling rates at ambient and higher pressures were $0.95 \mathrm{~K} / \mathrm{min}$, and the rate of increasing pressure was $10 \mathrm{MPa} / \mathrm{min}$.

Dielectric loss peaks after two different paths are reported for the secondary processes of the six systems in Figure 6 . The spectra are vertically shifted to have the same value of permittivity at the maximum. The frequency of maximum of the $\beta$-peak of PPGE shows a big change after two different thermodynamic histories as well as that of PDE, PVAc, and DGBEA. Instead, the maximum frequencies of $\beta$ relaxation of BMPC and of $\gamma$-relaxation of PPG400 are not 
TABLE 1: Parameters describing the pressure dependence of secondary processes.

\begin{tabular}{|c|c|c|c|c|}
\hline & $T(\mathrm{~K})$ & $d \log \left(\nu_{\max }\right) / d P$ & Activation volume $(\mathrm{mL} / \mathrm{mol})$ & $\log \left(\nu_{\max }\right)_{P=0}$ \\
\hline \multirow{6}{*}{ PPGE } & 293 & $(26.1 \pm 1) 10^{-4}$ & $14.7 \pm 0.5$ & $4.92 \pm 0.09$ \\
\hline & 283 & $(29.6 \pm 1) 10^{-4}$ & $16.1 \pm 0.5$ & $4.61 \pm 0.07$ \\
\hline & 268 & $(352 \pm 2) 10^{-5}$ & $18.2 \pm 0.1$ & $4.31 \pm 0.02$ \\
\hline & 263 & $(365 \pm 2) 10^{-5}$ & $18.5 \pm 0.1$ & $4.07 \pm 0.03$ \\
\hline & 253 & $(369 \pm 2) 10^{-5}$ & $18.0 \pm 0.1$ & $3.84 \pm 0.02$ \\
\hline & 240 & $(369 \pm 2) 10^{-5}$ & $17.4 \pm 0.1$ & $3.5 \pm 0.05$ \\
\hline \multirow{3}{*}{ PDE } & 293 & $(35.1 \pm 3) 10^{-4}$ & $21.3 \pm 0.7$ & $4.2 \pm 0.1$ \\
\hline & 276 & $(36.4 \pm 1) 10^{-4}$ & $20.8 \pm 0.5$ & $4.1 \pm 0.1$ \\
\hline & 253 & $(40.8 \pm 1) 10^{-4}$ & $21.4 \pm 0.5$ & $3.1 \pm 0.1$ \\
\hline \multirow{3}{*}{ BMPC } & 260 & $(102 \pm 3) 10^{-5}$ & $5.5 \pm 0.1$ & $2.5 \pm 0.01$ \\
\hline & 246 & $(157 \pm 2) 10^{-5}$ & $8.0 \pm 0.1$ & $2.47 \pm 0.01$ \\
\hline & 233 & $(135 \pm 4) 10^{-5}$ & $6.5 \pm 0.1$ & $2.1 \pm 0.06$ \\
\hline PPG400 & 238 & $(65 \pm 4) 10^{-5}$ & $3.2 \pm 0.1$ & $8.1 \pm 0.1$ \\
\hline \multirow{6}{*}{ PVAC } & 342 & $(174 \pm 3) 10^{-5}$ & $12.3 \pm 0.2$ & $6.71 \pm 0.01$ \\
\hline & 323 & $(218 \pm 4) 10^{-5}$ & $14.6 \pm 0.2$ & $6.5 \pm 0.01$ \\
\hline & 302 & $(32.8 \pm 1) 10^{-4}$ & $20.6 \pm 0.6$ & $6.6 \pm 0.04$ \\
\hline & 282 & $(31.4 \pm 1) 10^{-4}$ & $19.6 \pm 0.6$ & $6.4 \pm 0.04$ \\
\hline & 263 & $(339 \pm 6) 10^{-5}$ & $18.5 \pm 0.3$ & $5.3 \pm 0.02$ \\
\hline & 244 & $(353 \pm 6) 10^{-5}$ & $17.8 \pm 0.3$ & $4.8 \pm 0.02$ \\
\hline \multirow{3}{*}{ DGBEA } & 293 & $(349 \pm 4) 10^{-5}$ & $21.2 \pm 0.2$ & $5.63 \pm 0.02$ \\
\hline & 283 & $(333 \pm 4) 10^{-5}$ & $19.5 \pm 0.2$ & $5.08 \pm 0.02$ \\
\hline & 252.9 & $(422 \pm 1) 10^{-5}$ & $22.1 \pm 0.1$ & $4.41 \pm 0.04$ \\
\hline
\end{tabular}

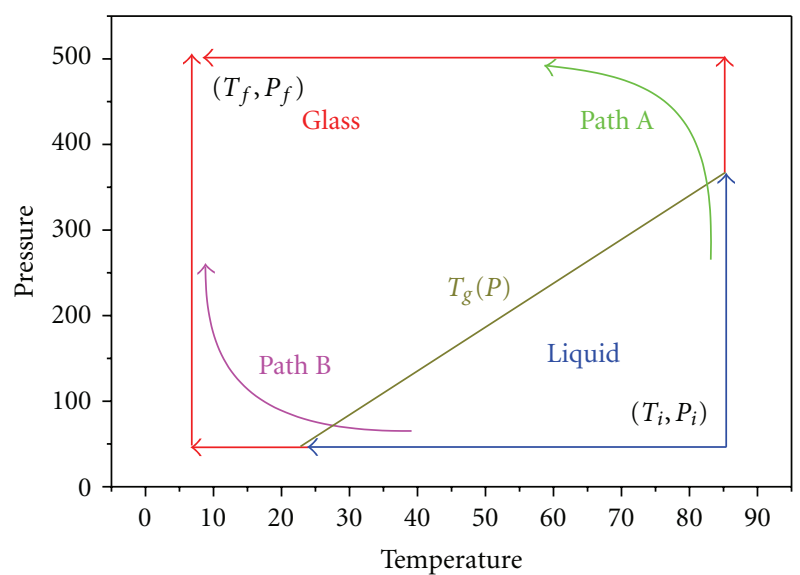

FIGURE 5: Schematic representation of the thermodynamic paths used in the experiment to vitrify the systems. In experiments, the starting point $\left(T_{i}, P_{i}\right)$ is in the liquid, and the final point $\left(T_{f}, P_{f}\right)$ in the glassy state.

affected by the preparation of the glass. In all systems where the secondary relaxation is affected by the thermodynamic history, the maximum frequency in glasses prepared along path $\mathrm{A}$ is lower than in glasses prepared along path $\mathrm{B}$.

Density variations can affect the secondary relaxation time. This is the case in our systems, since we observed that the relaxation frequency decreases on increasing pressure at fixed temperature, Figure 2. In fact, isothermal compression of a system corresponds to variations of density but not of thermal energy. The extent of the density effect on the
TABLE 2: Activation enthalpy $\left(\Delta H_{\beta}\right)$ and activation entropy, $\Delta S_{\beta}$, of the studied secondary processes at the different pressure values.

\begin{tabular}{lccc}
\hline & $P(\mathrm{MPa})$ & $\Delta H_{\beta}(\mathrm{KJ} / \mathrm{mol})$ & $\Delta S_{\beta}(\mathrm{J} / \mathrm{mol} / \mathrm{K})$ \\
\hline \multirow{2}{*}{ PPGE } & 500 & $63 \pm 2$ & $21.6 \pm 0.5$ \\
& 0.1 & $50.8 \pm 0.6$ & $21.3 \pm 0.2$ \\
PDE & 500 & $59.0 \pm 0.9$ & $13.0 \pm 0.3$ \\
& 0.1 & $51 \pm 1$ & $22 \pm 0.3$ \\
BMPC & 500 & $49.4 \pm 0.4$ & $3.0 \pm 0.2$ \\
& 0.1 & $47.0 \pm 0.5$ & $4.4 \pm 0.1$ \\
PVAc & 400 & $49 \pm 1$ & $11.3 \pm 0.2$ \\
& 300 & $49 \pm 2$ & $14.6 \pm 0.4$ \\
DGBEA & 0.1 & $39.1 \pm 0.1$ & $6.2 \pm 0.1$ \\
PPG400 & 0.1 & $52 \pm 1$ & $12.0 \pm 0.2$ \\
& 0.1 & $47.1 \pm 0.5$ & $16.6 \pm 0.2$ \\
\end{tabular}

secondary dynamics depends on the investigated system. For example, secondary relaxation in PDE and PPGE is more sensitive to pressure (larger values for activation volume) than that in BMPC and PPG400, Table 1. Glasses present different values of thermodynamic parameters, as density and expansivity, depending on their thermodynamic history. Since it is believed that such parameters affect the secondary relaxation dynamics, it is reasonable that also different relaxation frequencies can be observed for the secondary process. 


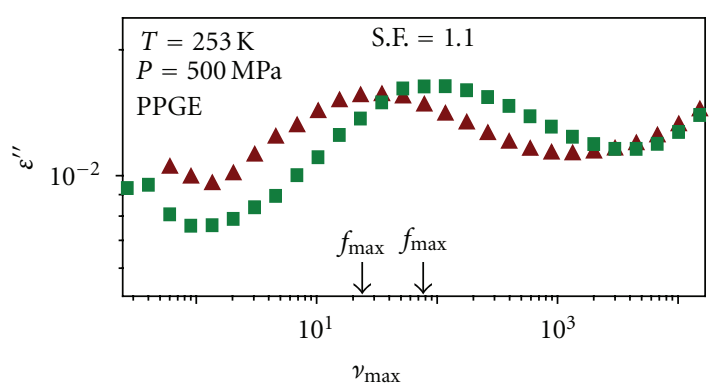

(a)

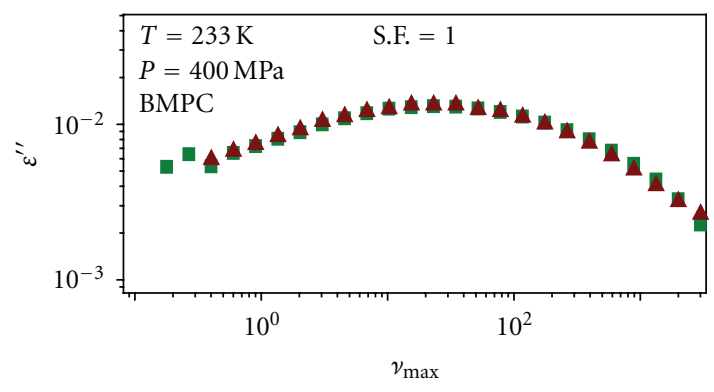

(c)

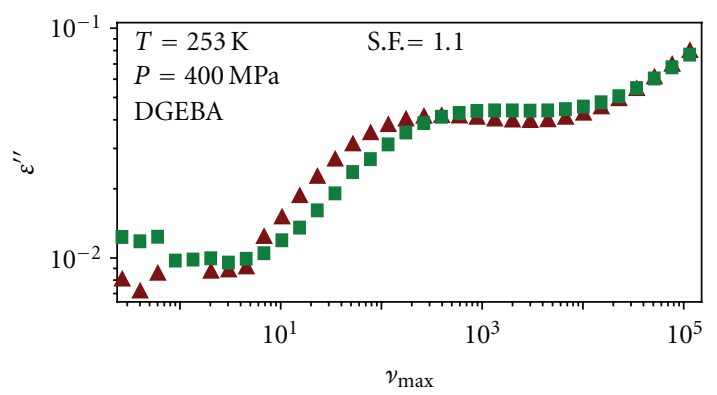

(e)

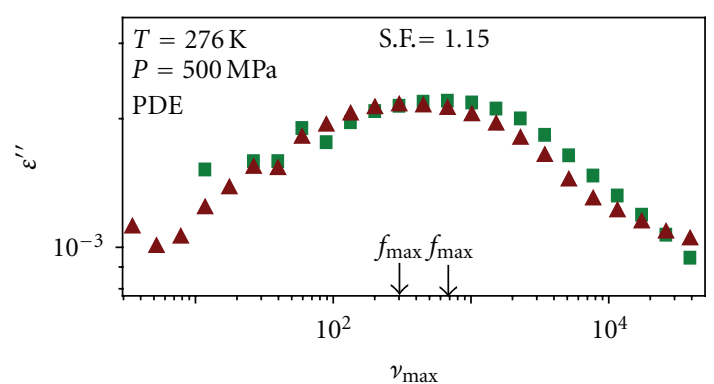

(b)

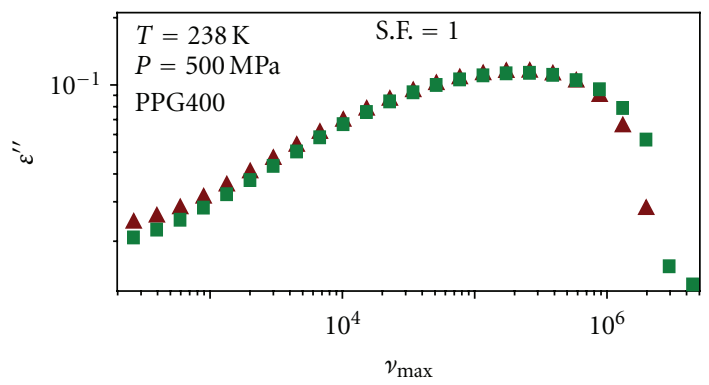

(d)

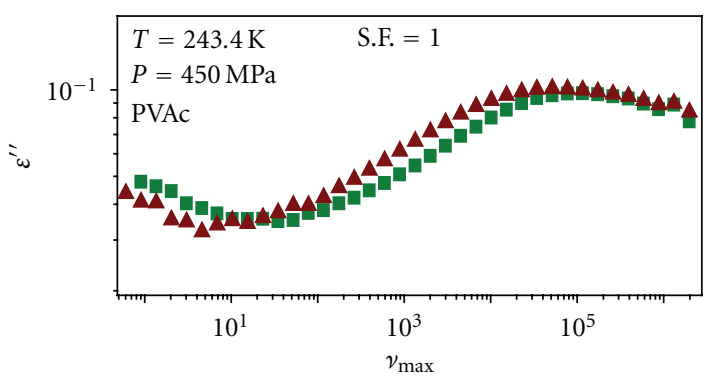

(f)

FIGURE 6: Loss spectra of the secondary relaxation measured after verification along path A (triangles) and B (squares) for (a) PPGE, (b) PDE, (c) BMPC, (d) PPG400, (e) DGBEA, and (f) PVAc. The spectra measured after verification along path A are vertically shifted by shift factor (S.F.) to obtain the same value of maxima loss as those measured after verification along path $B$.

TABle 3: Parameters of the thermodynamic paths used in this study to vitrify the systems; (Figure 5).

\begin{tabular}{lccccc}
\hline & \multicolumn{3}{c}{ Path A } & \multicolumn{2}{c}{ Path B } \\
& $T_{i}, P_{i}$ & $T_{f}, P_{f}$ & $T_{g}(P)$ & $T_{i}, P_{i}$ & $T_{f}, P_{f}$ \\
\hline \multirow{2}{*}{ PDE } & $353 \mathrm{~K}$ & $276 \mathrm{~K}$ & $353 \mathrm{~K}$ & $353 \mathrm{~K}$ & $276 \mathrm{~K}$ \\
& $0.1 \mathrm{MPa}$ & $500 \mathrm{MPa}$ & $240 \mathrm{MPa}$ & $0.1 \mathrm{MPa}$ & $500 \mathrm{MPa}$ \\
& $313 \mathrm{~K}$ & $253 \mathrm{~K}$ & $313 \mathrm{~K}$ & $313 \mathrm{~K}$ & $253 \mathrm{~K}$ \\
PPGE & $0.1 \mathrm{MPa}$ & $500 \mathrm{MPa}$ & $400 \mathrm{MPa}$ & $0.1 \mathrm{MPa}$ & $500 \mathrm{MPa}$ \\
& $293 \mathrm{~K}$ & $233 \mathrm{~K}$ & $293 \mathrm{~K}$ & $293 \mathrm{~K}$ & $233 \mathrm{~K}$ \\
BMPC & $0.1 \mathrm{MPa}$ & $400 \mathrm{MPa}$ & $290 \mathrm{MPa}$ & $0.1 \mathrm{MPa}$ & $400 \mathrm{MPa}$ \\
& $314 \mathrm{~K}$ & $238 \mathrm{~K}$ & $251 \mathrm{~K}$ & $314 \mathrm{~K}$ & $238 \mathrm{~K}$ \\
PPG400 & $0.1 \mathrm{MPa}$ & $500 \mathrm{MPa}$ & $500 \mathrm{MPa}$ & $0.1 \mathrm{MPa}$ & $500 \mathrm{MPa}$ \\
& $342 \mathrm{~K}$ & $302 \mathrm{~K}$ & $342 \mathrm{~K}$ & $342 \mathrm{~K}$ & $302 \mathrm{~K}$ \\
PVAc & $0.1 \mathrm{MPa}$ & $450 \mathrm{MPa}$ & $155 \mathrm{MPa}$ & $0.1 \mathrm{MPa}$ & $450 \mathrm{MPa}$ \\
& $293 \mathrm{~K}$ & $283 \mathrm{~K}$ & $293 \mathrm{~K}$ & $293 \mathrm{~K}$ & $283 \mathrm{~K}$ \\
DGBEA & $0.1 \mathrm{MPa}$ & $400 \mathrm{MPa}$ & $295 \mathrm{MPa}$ & $0.1 \mathrm{MPa}$ & $400 \mathrm{MPa}$ \\
\hline & & & & &
\end{tabular}

When a system is vitrified along path A, it is first compressed in the liquid state and then in the glassy state, whereas along the path B the system is compressed only in the glassy state, Figure 5. It is known that the compressibility of the liquid is larger than that of the glass. Moreover, compressing is usually more effective than cooling in increasing the density. According to these general considerations, one can expect that glasses prepared along path $\mathrm{A}$ are denser than those prepared along path B. Moreover, it is expected that denser systems present slower secondary relaxation, Figure 2. This idea is consistent with the observed results, Figure 6. In fact, in glassy PPGE, DGEBA, PVAc, and PDE prepared along path $\mathrm{A}$, the secondary relaxation is slower than in glasses prepared along path $\mathrm{B}$, and from the pressure dependence of the relaxation frequency, we know that this result can be related to a greater density of the latter sample. The question now is to understand if the density variation is the main factor determining the dependence of the secondary dynamics on thermodynamic history of the glass. Of course, 


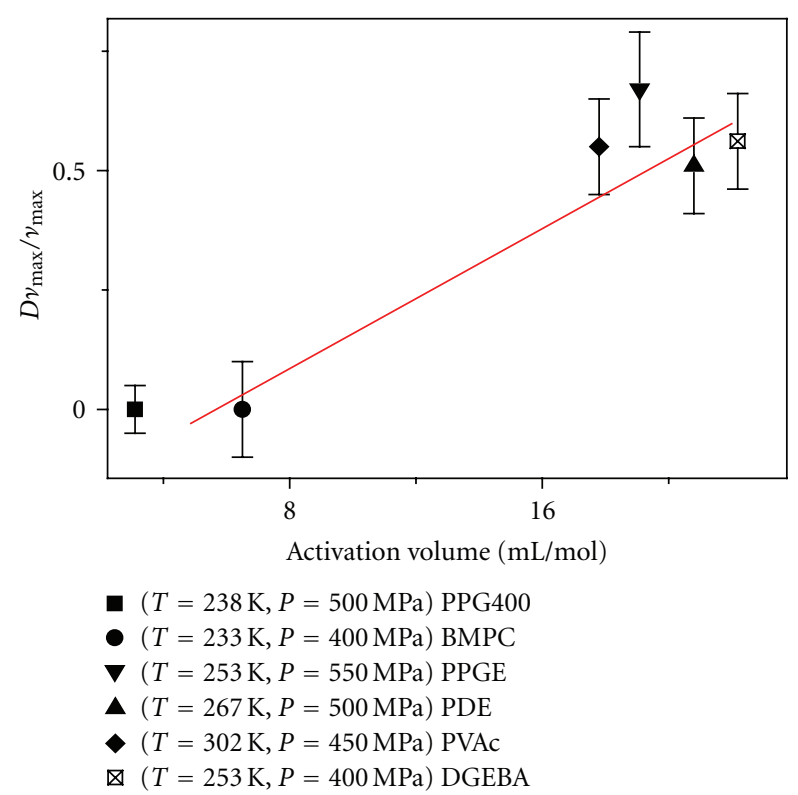

FIGURE 7: Normalized difference between frequencies of maximum of secondary relaxation peaks after two thermodynamic histories. The data are plotted as a function of the activation volume, $\Delta V_{\beta}$. The line is a guide for eyes.

for studying this issue density measurements are necessary, in connection to the dynamic ones. However, a rough estimation can be performed basing only on dynamics. In fact, the pressure dependence of secondary dynamics is determined in relation to its density dependence. If we assume that the expansivity of the four investigated systems is similar, then the different pressure dependences reflect different density dependences. In other words, with this approximation, we can use the value of activation volume as a rough estimation of the density dependence of the secondary relaxation. In Figure 7, we plot the relative variation of the frequency of the maximum loss peak, as calculated after the two paths. We observe that this quantity correlates with the activation volume in our systems: the bigger is the activation volume, the bigger is the effect of thermodynamic history on secondary relaxation. In other words, the larger is the pressure dependence of secondary relaxation frequency, the larger is the effect of thermodynamic history on it.

Figure 8 shows normalized difference between frequencies of maximum of secondary relaxation as a function of activation entropy, $\Delta S_{\beta}$, as it was estimated by (3) [31]. PPGE has big value of activation entropy, (21.7 \pm $0.1 \mathrm{~J} / \mathrm{mol}$ ), and the value of activation entropy for BMPC is small $(3.0 \pm 0.1 \mathrm{~J} / \mathrm{mol})$. Activation entropy values for PDE, DGBEA, and PVAc also intermediate between them. In Figure 8, it is observed that the normalized difference between frequencies of maximum of secondary relaxation increases with activation entropy, with the only exception of PPG400, as expected from the general idea that secondary relaxations with a more complex nature behave more similarly to the structural process. The case of PPG400 can be explained by considering the microscopic nature of its faster secondary process (that here considered). It is suggested that

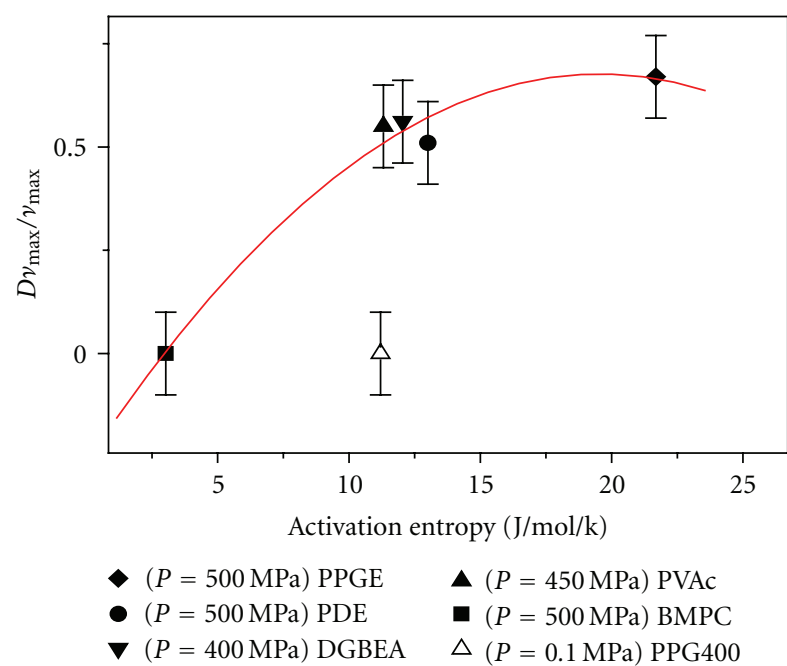

Figure 8: Normalized difference between frequencies of maximum of secondary relaxation peaks after two thermodynamic histories as a function of the activation Entropy, $\Delta S_{\beta}$. The line is a guide for the eyes.

its microscopic origin is related to the motion of the hydroxyl end groups, which can interact with each other through the formation of hydrogen bonds $[32,33]$. Due to this interaction, the hydroxyl group cannot move independently and for this reason the relaxation appears to be quite complex (high value of $\Delta S_{\beta}$ ). However, the hydroxyl group is very small and its motion is local not depending on the density of the material (weak pressure dependence). Our results showed that a greater sensitivity to the thermodynamic history is expected for secondary processes of intermolecular origin (JG) (e.g., PPGE and DGBEA) and less sensitivity is founded for non-JG relaxation (for example BMPC and PPG400). But also we can find some non-JG relaxation that they are senstive to the thermodynamic history (similar to the PDE and PVAc).

\section{Conclusions}

In this work, we performed several experiments with the aim of characterizing the dependence of the secondary relaxations on the thermodynamic history used to produce the glass. We investigated the effect that the thermodynamic path (pressure and temperature variations) followed in the vitrification procedure has on the characteristic frequency of the secondary relaxation (both for JG and non-JG relaxations), when measured in the glassy state at the same conditions of temperature and pressure. Secondary processes with larger activation volume and activation entropy are more dependent on the thermodynamic history. This result suggests that secondary relaxations reflecting complex motion are more sensitive to the preparation of the glass. The greater sensitivity could be related to variations of the local density of the environment where the process occurs: in fact, the dependence is more evident in those materials where the secondary process has more pressure, that is, density, and sensitivity. 


\section{References}

[1] P. G. Debenedetti and F. H. Stillinger, "Supercooled liquids and the glass transition," Nature, vol. 410, no. 6825, pp. 259$267,2001$.

[2] L. N. Hu and Y. Z. Yue, "Secondary relaxation behavior in a strong glass," Journal of Physical Chemistry B, vol. 112, no. 30, pp. 9053-9057, 2008.

[3] L. N. Hu and Y. Z. Yue, "Secondary relaxation in metallic glass formers: its correlation with the genuine Johari-Goldstein relaxation," Journal of Physical Chemistry C, vol. 113, no. 33, pp. 15001-15006, 2009.

[4] G. P. Johari and M. Goidstein, "Viscous liquids and the glass transition. II. Secondary relaxations in glasses of rigid molecules," The Journal of Chemical Physics, vol. 53, no. 6, pp. 2372-2388, 1970.

[5] D. Prevosto, S. Capaccioli, M. Lucchesi, P. A. Rolla, M. Paluch, and S. Pawlus, "Effect of thermodynamic history on secondary relaxation in glassy phenolphthalein-dimethyl-ether," Physical Review B, vol. 73, no. 10, Article ID 104205, pp. 1-5, 2006.

[6] E. Whalley and J. E. Bertie, "Optical spectra of orientationally disordered crystals. I. Theory for translational lattice vibrations," The Journal of Chemical Physics, vol. 46, no. 4, pp. 1264$1270,1967$.

[7] U. Buchenaii, N. Niicker, and A. J. Dianoux, "Neutron scattering study of the low-frequency vibrations in vitreous silica," Physical Review Letters, vol. 53, p. 2316, 1984.

[8] R. Mosseri and J. F. Sadoc, "Hierarchical structure of defects in non-crystalline sphere packings," Journal de Physique Lettres, vol. 45, no. 17, pp. 827-832, 1984.

[9] K. S. Evstropyev and E. A. Porai-Koshits, "Discussion on the modern state of the crystallite hypothesis of glass structure," Journal of Non-Crystalline Solids, vol. 11, no. 2, pp. 170-172, 1972.

[10] P. W. Anderson, B. I. Halperin, and C. M. Varma, "Anomalous low-temperature thermal properties of glasses and spin glasses," Philosophical Magazine, vol. 25, no. 1, pp. 1-9, 1972.

[11] R. Casalini, S. Capaccioli, M. Lucchesi et al., "Effect of pressure on the dynamics of glass formers," Physical Review E, vol. 64, no. 4, Article ID 041504, pp. 1-4, 2001.

[12] S. Corezzi, M. Beiner, H. Huth et al., "Two crossover regions in the dynamics of glass forming epoxy resins," Journal of Chemical Physics, vol. 117, no. 5, pp. 2435-2448, 2002.

[13] R. Casalini, M. Paluch, and C. M. Roland, "Dynamic crossover in supercooled liquids induced by high pressure," Journal of Chemical Physics, vol. 118, no. 13, pp. 5701-5703, 2003.

[14] S. Kahle, J. Gapinski, G. Hinze, A. Patkowski, and G. Meier, "A comparison of relaxation processes in structurally related van der Waals glass formers: the role of internal degrees of freedom," Journal of Chemical Physics, vol. 122, no. 7, Article ID 074506, pp. 1-10, 2005.

[15] S. Hensel-Bielowka and M. Paluch, "Origin of the highfrequency contributions to the dielectric loss in supercooled liquids," Physical Review Letters, vol. 89, no. 2, Article ID 025704, pp. 1-4, 2002.

[16] R. Casalini and C. M. Roland, "Scaling of the supercooled dynamics and its relation to the pressure dependences of the dynamic crossover and the fragility of glass formers," Physical Review B, vol. 71, no. 1, Article ID 014210, 10 pages, 2005.

[17] R. Casalini, M. Paluch, and C. M. Roland, "Influence of molecular structure on the dynamics of supercooled van der Waals liquids," Physical Review E, vol. 67, no. 3, Article ID 031505, p. 5, 2003.

[18] M. Paluch, C. M. Roland, R. Casalini, G. Meier, and A. Patkowski, "The relative contributions of temperature and volume to structural relaxation of van der Waals molecular liquids," Journal of Chemical Physics, vol. 118, no. 10, pp. 45784582, 2003.

[19] K. Grzybowska, A. Grzybowski, J. Zioło, M. Paluch, and S. Capaccioli, "Dielectric secondary relaxations in polypropylene glycols," Journal of Chemical Physics, vol. 125, no. 4, Article ID 044904, 2006.

[20] J. E. McKinney and R. Simha, "Configurational thermodynamic properties of polymer liquids and glasses. Poly(vinyl acetate). II," Macromolecules, vol. 9, no. 3, pp. 430-441, 1976.

[21] G. Dlubek, D. Kilburn, and M. A. Alam, "Temperature and pressure dependence of $\alpha$-relaxation and free volume in poly(vinyl acetate)," Macromolecular Chemistry and Physics, vol. 206, no. 8, pp. 818-826, 2005.

[22] M. Tyagi, A. Aleg, and J. Colmenero, "Broadband dielectric study of oligomer of poly(vinyl acetate): a detailed comparison of dynamics with its polymer analog," Physical Review E, vol. 75, no. 6, Article ID 061805, 2007.

[23] L. Goitiandia and A. Alegría, "Physical aging of poly(vinyl acetate). A thermally stimulated depolarization current investigation," Journal of Non-Crystalline Solids, vol. 287, no. 1-3, pp. 237-241, 2001.

[24] D. Prevosto, S. Capaccioli, S. Soheil, K. Kessairi, M. Lucchesi, and P. A. Rolla, "Secondary dynamics in glass formers: relation with the structural dynamics and the glass transition," Journal of Non-Crystalline Solids, vol. 353, no. 47-51, pp. 4278-4282, 2007.

[25] S. Capaccioli, D. Prevosto, K. Kessairi, M. Lucchesi, and P. Rolla, "Relation between the dispersion of $\alpha$-relaxation and the time scale of $\beta$-relaxation at the glass transition," Journal of Non-Crystalline Solids, vol. 353, no. 41-43, pp. 3984-3988, 2007.

[26] K. Grzybowska, A. Grzybowski, J. Zioło, M. Paluch, and S. J. Capaccioli, "Dielectric secondary relaxations in polypropylene glycols," Journal of Chemical Physics, vol. 125, no. 4, Article ID 044904, 2006.

[27] D. Prevosto, S. Sharifi, S. Capaccioli, P. A. Rolla, S. HenselBielowka, and M. Paluch, "New experimental evidence about secondary processes in phenylphthalein- dimethylether and 1, 1' -bis (p -methoxyphenyl)cyclohexane," Journal of Chemical Physics, vol. 127, no. 11, Article ID 114507, 2007.

[28] M. Paluch, R. Casalini, S. Hensel-Bielowka, and C. M. Roland, "Effect of pressure on the $\alpha$ relaxation in glycerol and xylitol," Journal of Chemical Physics, vol. 116, no. 22, pp. 9839-9844, 2002.

[29] J. K.N. Sharma and K. K. Jain, "Effect of pressure and temperature on the secondary relaxation in vinyl chloridevinyl acetate copolymer films by the PSD and TSD current techniques," Journal of Physics D, vol. 15, no. 2, pp. 337-342, 1982.

[30] G. Kasper and A. Reiser, "A volumetric and dielectric study of m-fluoroaniline under pressure," Journal of Non-Crystalline Solids, vol. 352, no. 42-49, pp. 4900-4904, 2006.

[31] H. W. Starkweather Jr., "Simple and complex relaxations," Macromolecules, vol. 14, no. 5, pp. 1277-1281, 1981.

[32] R. Casalini and C. M. Roland, "Excess wing in the dielectric loss spectra of propylene glycol oligomers at elevated pressure," Physical Review B, vol. 69, no. 9, Article ID 094202, pp. $1-7,2004$

[33] K. Wiemers and J. F. Kauffman, "Dielectric friction and rotational diffusion of hydrogen bonding solutes," Journal of Physical Chemistry A, vol. 104, no. 3, pp. 451-457, 2000. 

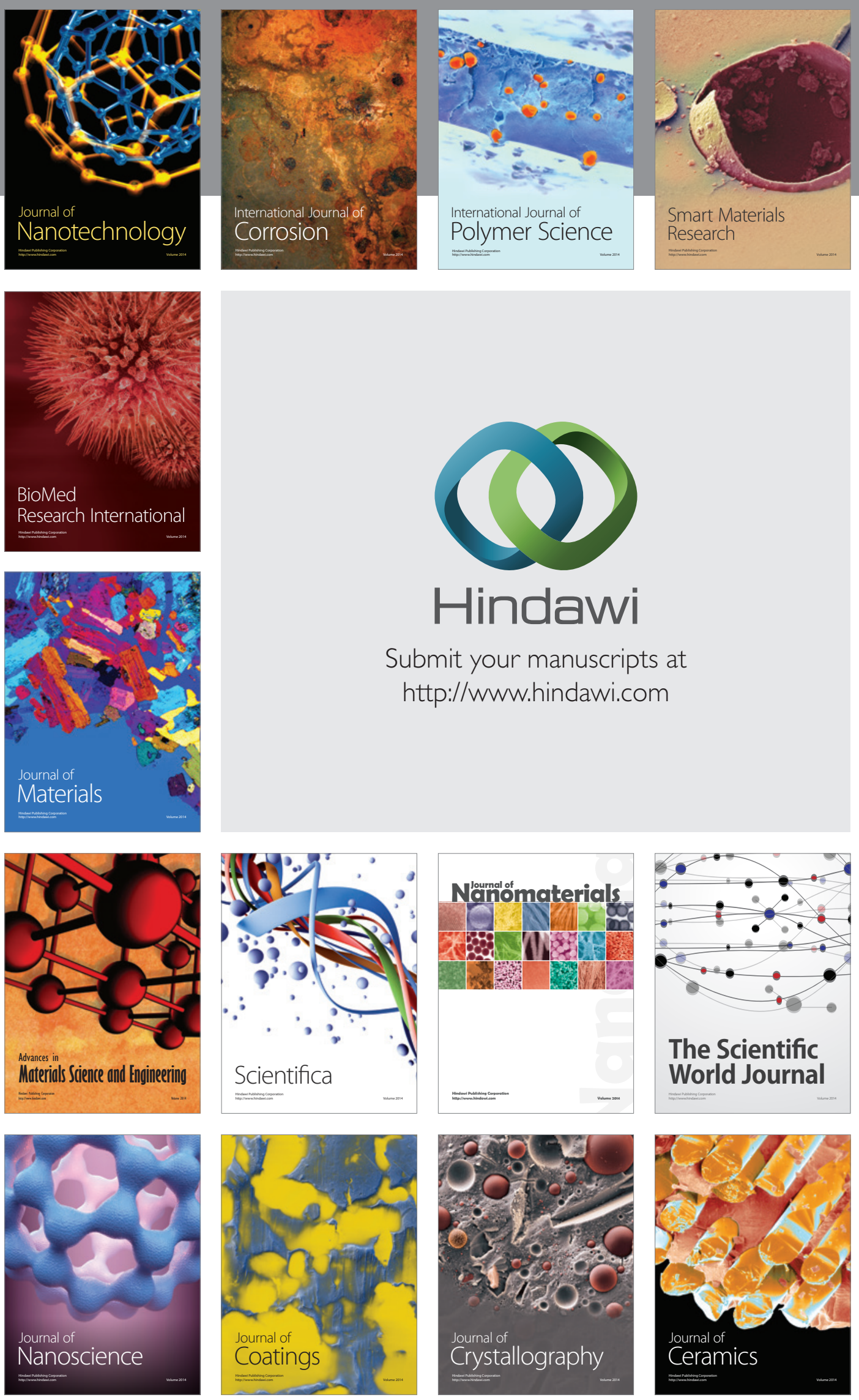

The Scientific World Journal

Submit your manuscripts at

http://www.hindawi.com

\section{World Journal}

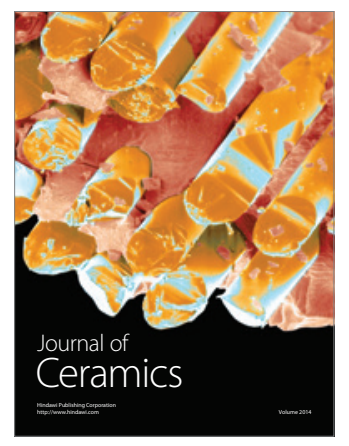

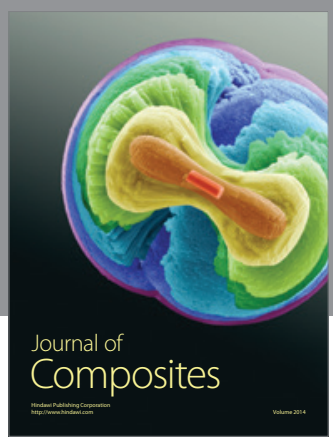
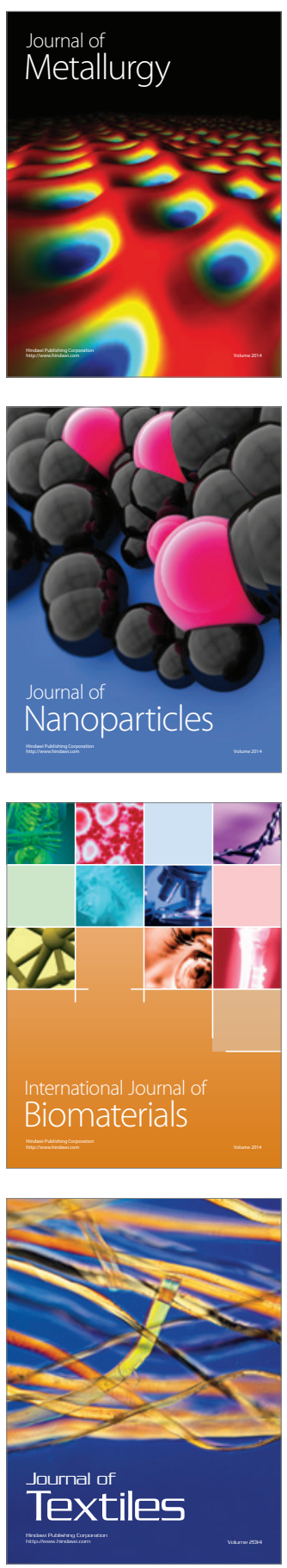